\title{
Buyang Huanwu Tang improves denervation-dependent muscle atrophy by increasing ANGPTL4, and increases NF- $\mathrm{KB}$ and MURF1 levels
}

\author{
LAN ZHOU $^{1}$, YUFANG HUANG $^{2}$, HUI XIE $^{3}$ and XIAOYUN MEI ${ }^{1}$ \\ ${ }^{1}$ Basic Theory of Traditional Chinese Medicine Staff Room, Basic Medical College; ${ }^{2}$ Pathological Staff Room, \\ Basic Medical College; ${ }^{3}$ Pharmacological Staff Room, School of Pharmacy, Nanjing University of \\ Traditional Chinese Medicine, Nanjing, Jiangsu 210023, P.R. China
}

Received November 2, 2016; Accepted July 13, 2017

DOI: $10.3892 / \mathrm{mmr} .2017 .8306$

\begin{abstract}
Denervated-dependent skeletal muscle atrophy (DSMA) is a disorder caused by the peripheral neuro-disconnection of skeletal muscle. The current study aimed to investigate the molecular mechanism and potential therapeutic strategies for the DSMA. A DSMA rat model was established. A lentiviral vector expressing small interfering RNA (siRNA) targeting angiopoietin-like protein 4 (ANGPTL4) was generated and injected into the rats that were also treated with Buyang Huanwu Tang (BYHWT). Reverse transcription-quantitative polymerase chain reaction was performed to examine ANGPTL4 mRNA expression in anterior cervical muscle samples. Western blot assay was used to evaluate ANGPTL4, nuclear factor- $\kappa \mathrm{B}(\mathrm{NF}-\kappa \mathrm{B})$ and muscle RING-finger protein-1 (MURF1) expression. The ultrastructure of muscle tissues was viewed using transmission electron microscopy. The cell apoptosis in muscle tissues was detected using the terminal deoxynucleotidyl transferase dUTP nick end labeling. The results indicated that BYHWT treatment increased ANGPTL4 mRNA and protein levels in muscle tissues. The suppression of ANGPTL4 using siRNA significantly increased inflammatory cells compared with the control siRNA group. BYHWT protected the ultrastructure muscle tissues and inhibited cell apoptosis in the DSMA model. The protective effect of BYHWT protected may be mediated by increased expression of NF- $\mathrm{\kappa B}$ p65 and MURF1. In conclusion, BYHWT may improve denervation-dependent muscle atrophy by increasing ANGPTL4 expression, involving NF- $\mathrm{KB}$ and MURF1 signaling.
\end{abstract}

Correspondence to: Dr Xiaoyun Mei, Basic Theory of Traditional Chinese Medicine Staff Room, Basic Medical College, Nanjing University of Traditional Chinese Medicine, 138 Xianlin Road, Nanjing, Jiangsu 210023, P.R. China

E-mail: meixxyudr@outlook.com

Key words: denervated-dependent skeletal muscle atrophy, angiopoietin-like protein 4, Buyang Huanwu Tang, small interfering RNA

\section{Introduction}

Denervated-dependent skeletal muscle atrophy (DSMA) is a disorder caused by the peripheral neuro-disconnection of skeletal muscle $(1,2)$. The skeletal muscle atrophy (SMA) is triggered by pharmacological drug treatment, certain diseases (including cancer, end stage pulmonary disorders and heart failure) (3) and traumatic peripheral nerve injury, all of which can lead to the immediate loss or damage of the voluntary contractile function of the skeletal muscle (4). The muscle denervation mainly occurs following several clinical syndromes, including degenerative disc disorder, diabetic neuropathy, alcoholic neuropathy, trauma, spinal muscular atrophy, viral infection and amyotrophic lateral sclerosis $(2,5)$. Thus, when the above pathogenic factors that cause SMA and the muscle denervation are combined, the DSMA occurs, and ultimately results in irreversible muscle dysfunction $(6,7)$. Although DSMA has become prevalent in recent years, the molecular mechanism is elusive and there is no complete therapeutic strategy, which prevents the development of the pharmacological therapies for used in the clinic.

Recently, a study reported that angiopoietin-like protein 4 (ANGPTL4) is secreted by the human forearm muscle after eating a meal high in saturated fatty acids (8). ANGPTL4 is also thought to be important mediators of muscle atrophy, and to have an important role in muscle and wound repair (9). However, whether ANGPTL4 has a key role in DSMA and the mechanism by which denervation causes ANGPTL4 changes have not been fully investigated.

Buyang Huanwu Tang (BYHWT) has been used for treating hemiplegia, and is composed of Radix astragali, Semen Persicae, Carthami flos, Radix Paeoniae Rubra, Rhizoma Chuanxiong, Radix Angelicae Sinensis and Pheretima (10). The BYHWT has been demonstrated to improve the blood circulation, inhibit fibroblast proliferation and decrease liquefaction and wallerian degeneration, as well as enhance nerve cell growth (11). Therefore, it is speculated that the BYHWT may participate in the pathogenesis of DSMA.

The current study aimed to investigate the role of BYHWT in the development of DSMA and the mechanism that underlines the ANGPTL4 changes in a DSMA model. Thus, an 
ANGPTL-4-targeting siRNA was generated and used in an established DSMA rat model to determine the role and mechanism ANGPTL4 in the pathogenesis of DSMA. The findings demonstrated that ANGPTL4 has an important role in DSMA via triggering of nuclear factor $-\kappa \mathrm{B}(\mathrm{NF}-\kappa \mathrm{B})$ and muscle RING-finger protein-1 (MURF1) pathways.

\section{Materials and methods}

Reagents and materials. Male Sprague-Dawley (SD) rats (body weight, 230-250 g; 8-10 weeks old) were purchased from Beijing HFK Bioscience Co., Ltd., (Beijing, China). The rats were housed in the specific cages received free access to the water and food, and at a constant temperature. All of the experiments were performed according to the Guidelines of the Institutional Animal Care and approved by the Ethics Committee of the Nanjing University of Traditional Chinese Medicine (Nanjing, China).

The components of BYHWT, including Astragalus (480 g), Tangkuei tail (24 g), Paeoniae (20 g), Pheretima (12 g), Rhizoma Chuanxiong (12 g), Flos Carthami Tinctorii (12 g) and peach seeds (12 g), were purchased from Beijing Tong Ren Tang Co., Ltd. (Beijing China). The above Chinese medicine components were mixed together and obtained by boiling to a final volume of $1,500 \mathrm{ml}$ (supplementing with the distilled water), which was assigned as the BYHWT stock solution for the subsequent experiments. The chemical reagents, including $\mathrm{K}_{2} \mathrm{HPO}_{4}, \mathrm{~K}_{3} \mathrm{PO}_{4}$ and $\mathrm{MgCl}_{2}$, were purchased from Kelong Co., Ltd. (Chengdu, China).

Establishment of DSMA rat model. SD rats $(\mathrm{n}=8)$ were used to establish the DSMA model. Meanwhile, the other eight rats were selected as the sham operation group (Sham group). The rats were fixed in the prone position during the surgical process and then the rats were anesthetized by injecting intraperitoneally with $7 \%$ chloral hydrate at the concentration of $0.5 \mathrm{ml} / 100 \mathrm{~g}$. The surgery was conducted only on the left side of lower limb by cutting at the dorsolateral skin incision. Then, between the gluteus muscle and the biceps femoris, the common peroneal nerve was exposed, which was also separated and extracted from the connective tissues in the surrounding area, and exposing $\sim 1.5 \mathrm{~cm}$ of common peroneal nerve. The two ends of the common peroneal nerve were turned by $\sim 180^{\circ}$. In order to prevent the reconnection between the nerves, the nerves were sewn on the muscle membranes by using a 9-0 non-destructive wound suture (nylon). The DSMA model has been successfully established according to previously published studies $(3,4,6)$. For the sham group, the left sciatic nerves were only mildly exposed and separated from the surrounding tissues, and were not sewn by the 9-0 non-destructive wound suture.

Lentiviral vectors expressing ANGPTL4-siRNA. The high titer lentiviral vectors, which deliver the ANGPTL4-specific small interfering RNA (siRNA) and control siRNA were generated according to the previous published reports $(12,13)$. The following siRNA sequences targeting the ANGPTL-4 was designed and assigned as ANGPTL4-siRNA: Sense, 5'-AAAGCTGCAAGATGACCTCAGATGGAGGCTG-3'; and anti-sense, 5'-AAAAGGCTTAAGAAGGGAATCTTCTGG
AAGAC-3'. The following control siRNA sequences was also designed and assigned as CON-siRNA: Sense, 5'-AAAGCT GTCTTCAAGATTGATATCGAAGACTA-5'; and anti-sense, 5'-AAAATAGTCTTCGATATCAAGCTTGAAGACA-3'. Then, the ANGPTL4-siRNA and CON-siRNA were cloned into the lentiviral vectors to synthesize the ANGPTL-4 siRNA lentiviral vector, which was used to infect the DSMA rat models.

Trial grouping and treatment. In this study, the DSMA rat models were divided into three groups, including DSMA + saline, assigned as model group, DSMA + CON-siRNA lentiviral vector [tail vein injection with $100 \mu \mathrm{l}$ (100 multiplicity of infection) at 14th day] + BYHWT (twice diluted BYHWT stock solution, $2 \mathrm{ml}$ every day per os), assigned as Con-siRNA group, and DSMA + ANGPTL4-siRNA lentiviral vector (tail vein injection with $100 \mu 1$ at 14th day) + BYHWT (twice diluted BYHWT stock solution, $2 \mathrm{ml}$ every day), assigned as ANGPTL4-siRNA group.

The BYHWT was also divided into three groups, including high-dose BYHWT group (BYHWT-high, BYHWT stock solution diluted 2X), moderate-dose BYHWT group (BYHWT-moderate, BYHWT stock solution diluted 4X) and low-dose BYHWT group (BYHWT-low, BYHWT stock solution diluted $8 \mathrm{X})$.

Reverse transcription-quantitative polymerase chain reaction (RT-qPCR) gene expression assay. Total RNA was extracted from the anterior cervical muscle samples using TRIzol reagent (Thermo Fisher Scientific, Inc., Waltham, MA, USA). Briefly, the sciatic nerves were lysed by using $1 \mathrm{ml}$ TRIzol. Subsequently, the RNAs were extracted by using $200 \mu 1$ chloroform (Western Biotech. Co., Ltd., Chongqing, China), and the supernatant was stored for the further experiments. Then, the RNAs were precipitated by using isopropanol (1 ml), followed with washing in $70 \%$ ethanol $(1 \mathrm{ml}$, twice). Finally, the RNA precipitations were diluted in the diethylpyrocarbonate-treated water. The cDNA was synthesized by using the RT method in a $10 \mu \mathrm{l}$ system including oligo dT, total RNA, RT buffer (5X), random primer mix, RNase inhibitor, RT enzyme mix (Beyotime Institute of Biotechnology, Shanghai, China) and $\mathrm{ddH}_{2} \mathrm{O}$. The RT-qPCR process was performed at $37^{\circ} \mathrm{C}$ for $10 \mathrm{~min}$, followed by $95^{\circ} \mathrm{C}$ for $5 \mathrm{~min}$. Amplification of ANGPTL4 was performed by using the synthesized cDNA as the template. The primers for ANGPTL4 (129 bp) were as follows: Forward, 5'-AGAAGTTGGAGATGCAGAGGGAC-3' and reverse, 5'-CCACAAGAGCACCATTGAGTGTAT-3'. The primers for the internal control $\beta$-actin (150 bp) were as follows: Forward, 5'-CCCATCTATGAGGGTTACGC-3' and reverse, 5'-TTTAATGTCACGCACGATTTC-3'. The total volume of the PCR system was a $10 \mu \mathrm{l}$ reaction, which included forward primer $(1 \mu \mathrm{l})$, reverse primer $(1 \mu \mathrm{l}), 2 \mathrm{X}$ SYBR-Green Mixture (4.5 $\mu$; Invitrogen; Thermo Fisher Scientific, Inc.), cDNA $(1 \mu \mathrm{l})$, and $\mathrm{ddH}_{2} \mathrm{O}(2.5 \mu \mathrm{l})$. The PCR conditions were as follows: $95^{\circ} \mathrm{C}$ for $10 \mathrm{sec}, 55^{\circ} \mathrm{C}$ for $40 \mathrm{sec}$ and $75^{\circ} \mathrm{C}$ for $30 \mathrm{sec}$. The RT-qPCR was performed on the FTC2000 fluorescent qPCR cycler for 40 cycles. Finally, the amplified products were loaded onto $1.5 \%$ agarose gels, and the images were analyzed by using the Quantity One image analysis software (version 4.6.5; Bio-Rad Laboratories, Inc., Hercules, CA, USA). The relative mRNA expression of targeting genes 
was normalized to the $\beta$-actin gene by using the comparative threshold cycle $\left(2^{-\Delta \Delta \mathrm{Cq}}\right)$ method (6).

Western blotting. The anterior cervical muscle samples were lysed by using the radioimmunoprecipitation assay lysis buffer (Sigma-Aldrich; Merck KGaA, Darmstadt, Germany), and centrifuged at $12,000 \mathrm{x}$ g for $30 \mathrm{~min}$. Then, the supernatant $(50 \mu \mathrm{g})$, the protein concentration was quantified by using the bicinchoninic assay method according to the manufacturer's protocol (Beyotime Institute of Biotechnology) were separated by using $10 \%$ SDS-PAGE, and then were transferred on to a polyvinylidene fluoride membrane (Dupont, Wilmington, DE, USA) by using the Trans-Blot SD cell instrument (Bio-Rad Laboratories, Inc.). The membrane was blocked by using $5 \%$ non-fat milk at room temperature for $2 \mathrm{~h}$, followed by incubation with the rabbit anti-rat ANGPTL4 polyclonal antibody (1:2,000; cat. no. AF3485; R\&D Systems, Inc., Minneapolis, MN, USA), rabbit anti-rat NF- $\kappa$ B p65 polyclonal antibody (1:1,000; cat. no. MAB50781; R\&D Systems, Inc.), mouse anti-rat MURF1 monoclonal antibody (1:3,000; cat. no. AF5366; R\&D Systems, Inc.) and the rabbit anti-rat actin (internal control, cat. no. MAB1420; R\&D Systems, Inc.) polyclonal antibody at $4^{\circ} \mathrm{C}$ overnight. The membranes were washed by using the PBS Tween-20 (PBST) buffer three times for $5 \mathrm{~min}$. The membranes were incubated with the horseradish peroxidase (HRP)-labeled goat anti-rabbit IgG (1:1,000; cat. no. AQ132P; Sigma-Aldrich; Merck KGaA) and HRP-labeled goat anti-mouse IgG (1:1,000; cat. no. AP308P; Sigma-Aldrich; Merck $\mathrm{KGaA}$ ) for $60 \mathrm{~min}$ at $37^{\circ} \mathrm{C}$, and washed with PBST buffer three times for $5 \mathrm{~min}$. Finally, the enhanced chemiluminescence reagent (Pierce; Thermo Fisher Scientific, Inc.) was used to treat the membrane for $2 \mathrm{~min}$ in the dark. The relative grey density of the bands were analyzed by using the Labworks Analysis Software (version 4.5; UVP, Inc., Upland, CA, USA; www.uvp.com).

Transmission electron microscopy (TEM). Anterior cervical muscles tissues were fixed in $10 \%$ neutral buffered formalin for $15 \mathrm{~min}$ at $37^{\circ} \mathrm{C}$ and minced into the pieces of $1 \mathrm{~mm}^{3}$. Then, the pieces were immersed in $2.5 \%$ glutaraldehyde for $2 \mathrm{~h}$ at $37^{\circ} \mathrm{C}$ (dissolved into the $0.1 \mathrm{M}$ PBS, and adjusted to $\mathrm{pH}$ 7.4). The formalin-fixed tissues were embedded in paraffin and mounted on glass slides. Then, the tissues were stained with $0.5 \%$ periodic acid-Schiff reagent for $15 \mathrm{~min}$ at $37^{\circ} \mathrm{C}$, following the diastase treatment. The status of the coverslips was examined using a light microscope. The muscle tissues incubated in glutaraldehyde were rinsed thoroughly with PBS. The muscle tissues were continuously post-fixed in $2 \% \mathrm{OsO}_{4}$ at room temperature for $2 \mathrm{~h}$, and dehydrated and embedded Embed 812. The muscle tissues were embedded and polymerized overnight at $60^{\circ} \mathrm{C}$ in the flat embedding molds. The areas of interest were selected using $0.5 \%$ toluidine blue-stained sections $(0.5 \mu \mathrm{m})$ at room temperature for $15 \mathrm{~min}$. Then, ultrathin sections $(0.1-\mu \mathrm{m})$ were cut and mounted on the grids, and then stained using $2 \%$ uranyl acetate for $10 \mathrm{~min}$ at $37^{\circ} \mathrm{C}$. Finally, the sections were examined by using a Tecnal-10 TEM (Philips Medical Systems B.V., Eindhoven, The Netherlands).

Terminal deoxynucleotidyl transferase dUTP nick end labeling (TUNEL) assay. In order to observe the possible
DNA fragmentation in the anterior cervical muscle tissues, TUNEL was performed by using a TUNEL kit (Roche Diagnostics, Indianapolis, IN, USA) in the present study. In brief, the isolated anterior cervical muscle tissues were fixed with $4 \%$ paraformaldehyde for $10 \mathrm{~min}$ at room temperature in $\mathrm{NaH}_{2} \mathrm{PO}_{4}(0.1 \mathrm{M}$, adjusted to $\mathrm{pH}$ 7.4). Endogenous peroxidase activity in the anterior cervical muscle tissues were inactivated using $3 \% \mathrm{H}_{2} \mathrm{O}_{2}$ for $10 \mathrm{~min}$ at room temperature. The tissues were incubated with biotin-dUTP solution and incubated with the terminal deoxynucleotidyl transferase $(20 \mathrm{U} / \mu \mathrm{l})$ at $37^{\circ} \mathrm{C}$ for $1 \mathrm{~h}$. After the tissues were treated using the end-horseradish peroxidase, the anterior cervical muscle tissues were stained with the diaminobenzidine (final concentration of $0.05 \%$, at room temperature for $10 \mathrm{~min}$ ) and counterstained with the ethyl green (room temperature for $10 \mathrm{~min}$ ) to detect the stained and biotin-labeled cell nuclei. The apoptotic bodies stained brown and the cell nuclei were observed, and counted under the light microscope. For the quantitative analysis, the apoptosis index was evaluated as the percentage of apoptotic cells. The positively-stained nuclei were counted by at least three investigators and in at least three different fields.

Hematoxylin and eosin $(H \& E)$ staining assay. Anterior cervical muscles tissues were fixed with the $4 \%$ paraformaldehyde at room temperature for $24 \mathrm{~h}$. Then, the tissues were embedded in paraffin, and sectioned into $4-\mu \mathrm{m}$ thick sections. The paraffin was removed using the regular method (6), and the sections were use for the H\&E staining as previously described (6). The images were captured by the inverted fluorescence microscope (Olympus Corporation, Tokyo, Japan), and were analyzed by employing the Medical Image Analysis system (cat. no. HMIAS22000; Qianping Image Engineering Company, Tongji Medical University, Wuhan, China). The percentage of inflammatory cells was calculated as follows: (The number of inflammatory cells/the total number of cells) x100 (\%). The inflammatory cells were stained with the blue and the normal cells were without staining.

Statistical analysis. All data were analyzed by with the SPSS software 20.0 (IBM Corp., Armonk, NY, USA). The data are presented as the mean \pm standard deviation obtained from at least three independent experiments. The Student's t-test was performed to evaluate the statistical significance between the two groups, and the ANOVA test was performed to assess statistical significance among the multiple groups. $\mathrm{P}<0.05$ was considered to indicate a statistically significant difference.

\section{Results}

BYHWT treatment increases ANGPTL4 mRNA levels. In order to confirm the successful transfection of the ANGPTL-4 siRNA lentiviral vector to the rats, the ANGPTL4 mRNA and protein levels were observed by using RT-PCR and western blotting (Fig. 1). ANGPTL4 mRNA and protein levels in the ANGPTL4-siRNA group were significantly lower than the Con-siRNA group $(\mathrm{P}<0.05$; Fig. 1$)$, which suggests that the ANGPTL4 siRNA lentiviral vector was efficiently transfected and expressed. Additionally, the ANGPTL4 levels in the 
A
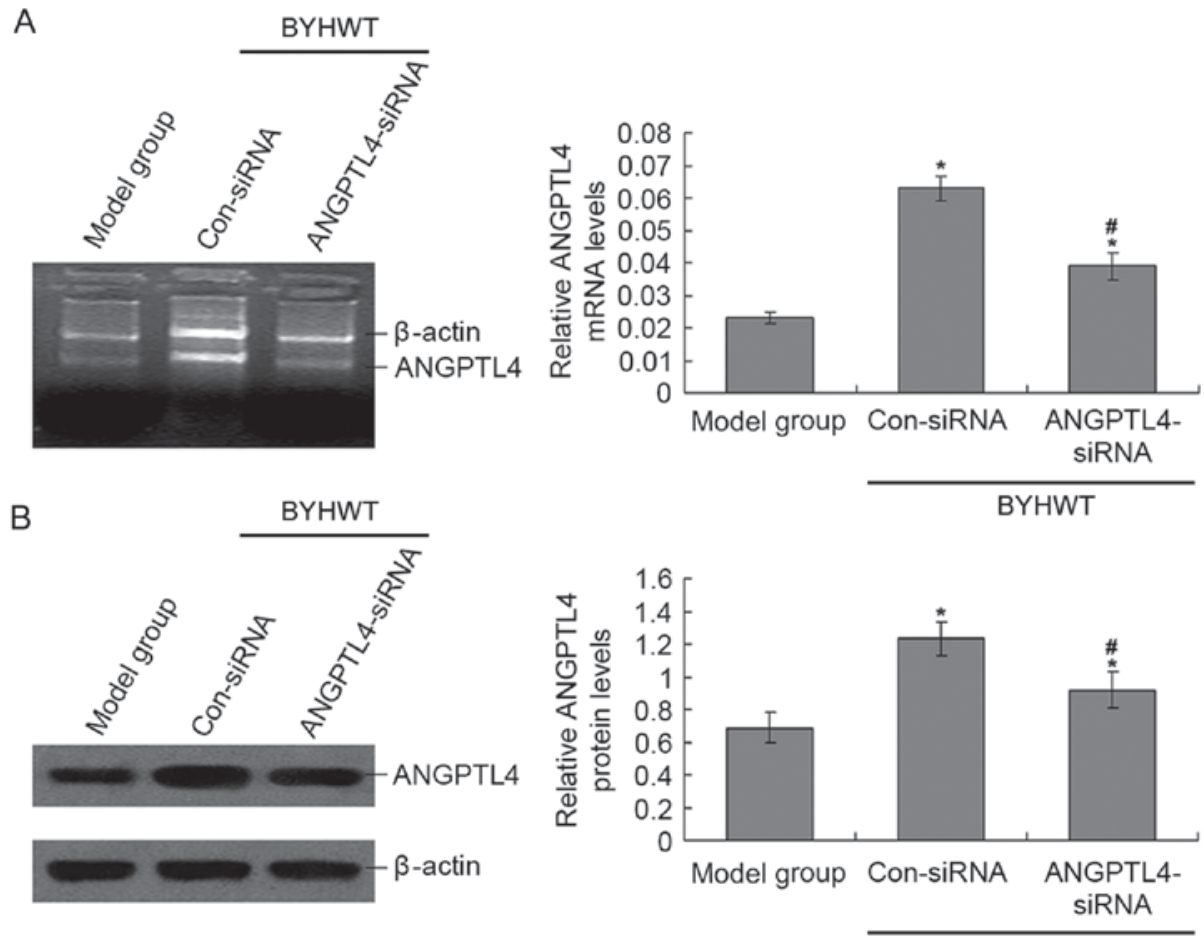

BYHWT

Figure 1. Detection of ANGPTL4 mRNA and protein expression in anterior cervical muscle samples. (A) ANGPTL4 mRNA expression was examined by using reverse transcription-quantitative polymerase chain reaction assay. (B) ANGPTL4 protein expression was examined using western blot assay. "P<0.05 vs. model group. ${ }^{\#} \mathrm{P}<0.05$ vs. Con-siRNA group. BYHWT, Buyang Huanwu Tang; Con, control; siRNA, small interfering RNA; ANGPTL4, angiopoietin-like protein 4.

A

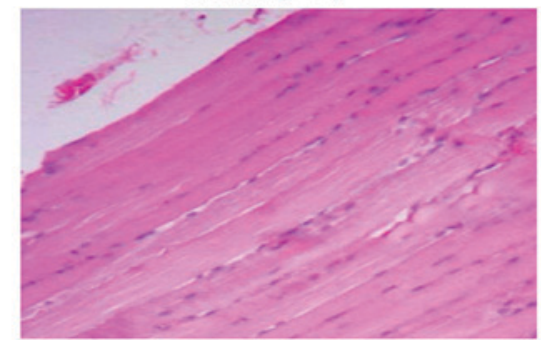

C

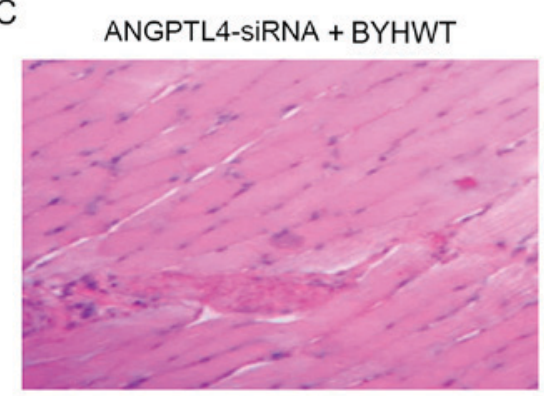

B

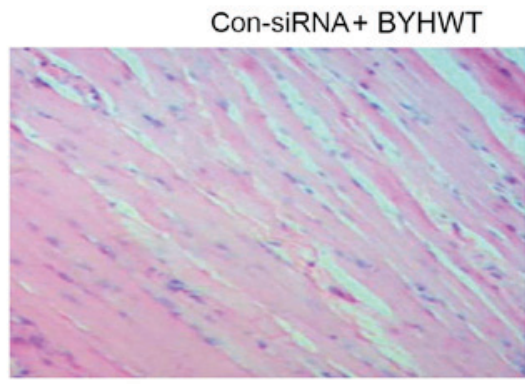

$\mathrm{D} z$

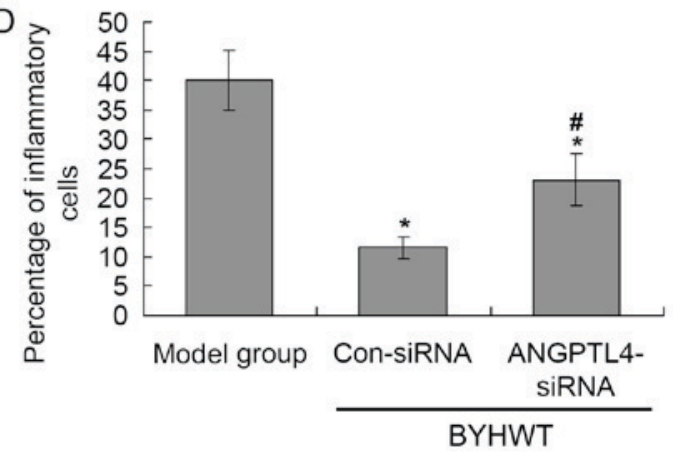

Figure 2. Hematoxylin and eosin staining of the anterior cervical muscle tissues. (A) Model group. (B) Con-siRNA group. (C) ANGPTL4-siRNA group. (D) Statistical analysis for the staining. "P<0.05 vs. model group. ${ }^{~} \mathrm{P}<0.05$ vs. Con-siRNA group. BYHWT, Buyang Huanwu Tang; Con, control; siRNA, small interfering RNA; ANGPTL4, angiopoietin-like protein 4.

Con-siRNA group were also significantly higher compared with the model group $(\mathrm{P}<0.05$; Fig. 1), which suggests that BYHWT significantly increased the levels of ANGPTL4. In the pre-experiment, the data of Sham rats compared with the normal rats were examined. The results demonstrated that there were no differences between the Sham rats and the normal rats (data not shown).

$H \& E$ staining. According to the H\&E staining results (Fig. 2), there were many inflammatory cells in the model group 

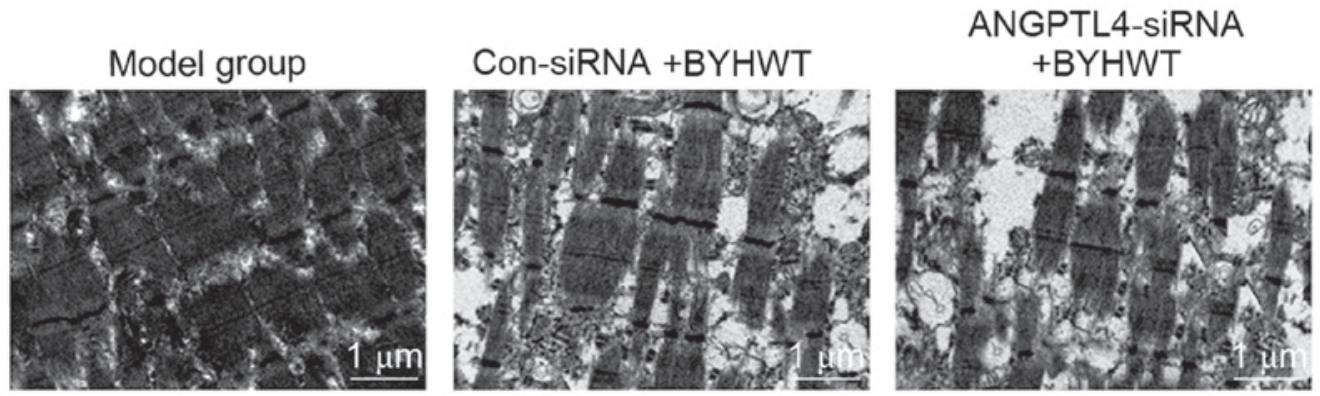

Figure 3. Transmission electron microscopy examination of the ultrastructure of muscle tissues. Representative images from model group, CON-siRNA group and ANGPTL4-siRNA group. BYHWT, Buyang Huanwu Tang; Con, control; siRNA, small interfering RNA; ANGPTL4, angiopoietin-like protein 4.
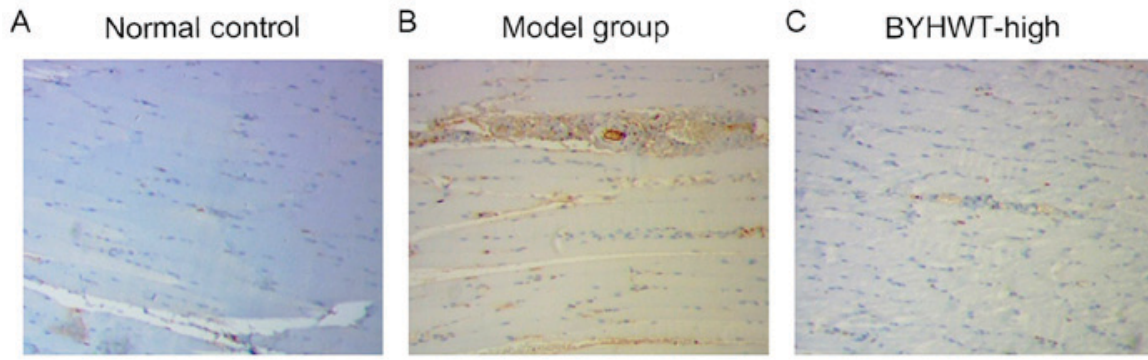

D BYHWT-moderate

E

F
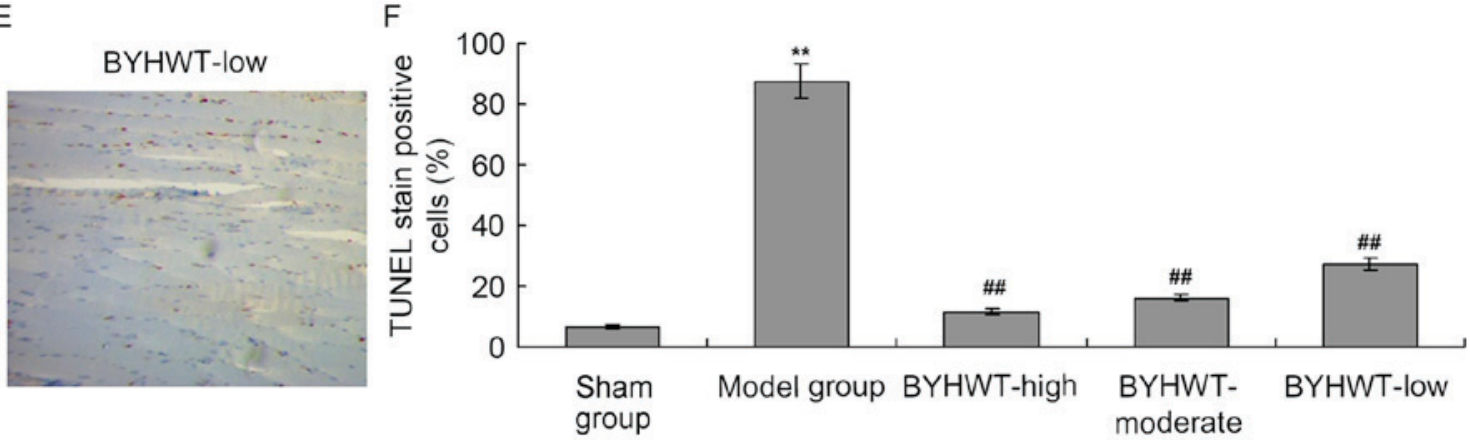

Figure 4. TUNEL staining for examining the cell apoptosis in anterior cervical muscle tissues. (A) Normal control. (B) Model group. (C) BYHWT-high group. (D) BYHWT-moderate. (E) BYHWT-low. (F) Quantification of TUNEL-positive cells. ${ }^{* *} \mathrm{P}<0.01$ vs. Sham group. ${ }^{\# \#} \mathrm{P}<0.01$ vs. model group. TUNEL, terminal deoxynucleotidyl transferase dUTP nick end labeling; BYHWT, Buyang Huanwu Tang.

(Fig. 2A), and BYHWT significantly decreased the levels of inflammatory cells compared with the model group $(\mathrm{P}<0.05$; Fig. 2B). Additionally, the suppression ANGPTL4 significantly increased the number of inflammatory cells compared with the Con-siRNA group ( $\mathrm{P}<0.05$; Fig. $2 \mathrm{C}$ and $\mathrm{D})$.

BYHWT protects the ultrastructure of muscle tissues. In the current study, TEM was performed to analyze the ultrastructure of the muscle tissues. The TEM findings demonstrated that the ultrastructure of muscle was seriously damaged in the model group (Fig. 3A). However, the BYHWT treatment (Con-siRNA group) observably improved the ultrastructure of muscle, including the muscle fibers being more ordered with fewer spaces between the fibers (Fig. 3B). Furthermore, the inhibition of ANGPTL4 (ANGPTL4-siRNA group) suppressed the effects of BYHWT on the ultrastructure of muscle tissues (Fig. 3C), which suggests that the ANGPTL-4 may participate in the repair of the muscle tissues.

BYHWT inhibits cell apoptosis in DSMA model. According to the TEM findings, the ultrastructure of muscle tissues was damaged in the DSMA model, therefore, the muscle tissues may be experiencing apoptosis. In the present study, the TUNEL assay was performed to investigate the apoptosis of the muscle tissue cells. The results indicated that there was obvious TUNEL staining in the model group compared with the normal control group ( $\mathrm{P}<0.01$; Fig. $4 \mathrm{~A}$ and $\mathrm{B})$. Additionally, the number of TUNEL-positive cells were also significantly decreased in the BYHWT-treated groups in a dose-dependent manner (from high to low; $\mathrm{P}<0.05$; Fig. 4C-F).

BYHWT reduces $N F-\kappa B$ p 65 and MURF1 expression in a DSMA model. In order to investigate the mechanism of the BYHWT-activated protective effects, the inflammatory signaling pathway factor, $N F-\kappa B$ p 65 , and the SMA-associated factor, MURF1, were detected by using the western blot assay. The results indicated that the establishment of the DSMA model increased the expression of $\mathrm{NF}-\kappa \mathrm{B}$ p65 and MURF1 compared with the normal rats $(\mathrm{P}<0.05$; Fig. 5). Furthermore, BYHWT treatment significantly decreased the levels of NF- $\kappa$ B p65 and MURF1 compared to the model group $(\mathrm{P}<0.05$; Fig. 5). Furthermore, the change 
A

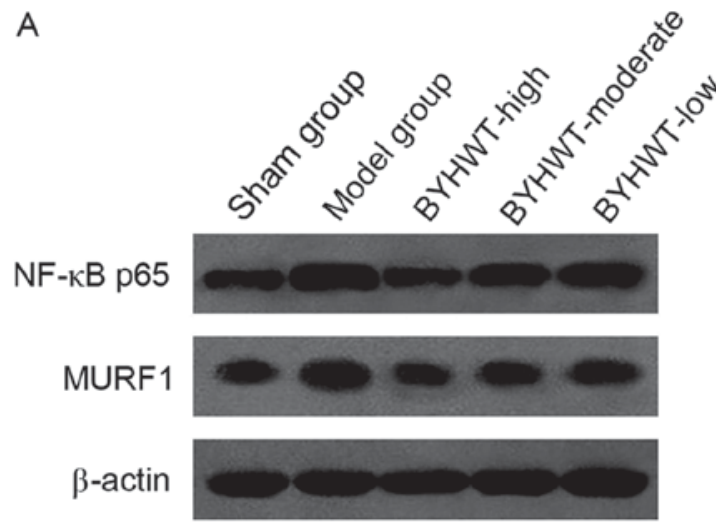

B

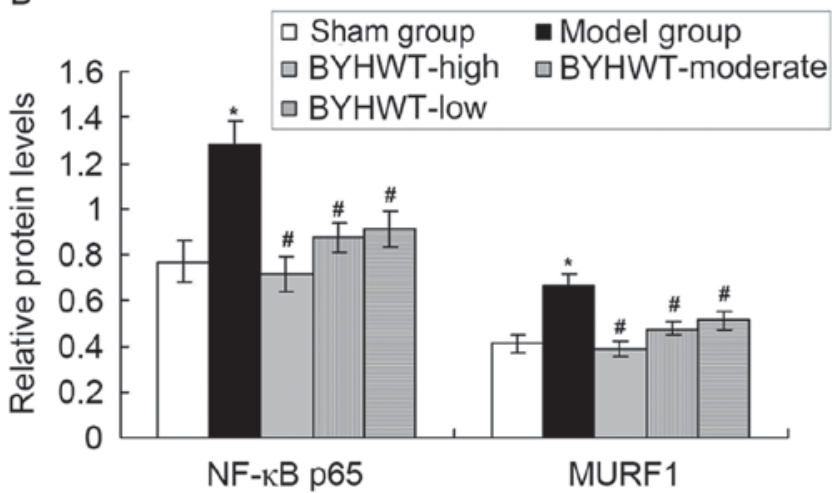

Figure 5. Examination of NF- $\mathrm{B}$ p65 and MURF1 protein expression in Model and BYHWT treatment group. (A) Western blot assay for NF- $\kappa$ B p65 and MURF1 expression. (B) Semi-quantification of protein expression. "P<0.05 vs. normal control group. " $\mathrm{P}<0.05$ vs. model group. BYHWT, Buyang Huanwu Tang; NF- $\kappa$ B, nuclear factor- $\kappa \mathrm{B}$; MURF1, muscle RING-finger protein-1.

in of NF- $\mathrm{B}$ p65 and MURF1 induced by BYHWT was dose-dependent.

\section{Discussion}

In the present study, it was demonstrated that ANGPTL4 expression is involved in the therapeutic effect of BYHWT in a DSMA model. BYHWT could improve the blood circulation, inhibit fibroblast proliferation and decrease liquefaction and Wallerian degeneration, as well as enhance the nerve cells growth according to the previously published studies $(11,14,15)$. ANGPTL4 is best known for its postprandial function in skeletal muscle lipid metabolism following a meal high in saturated fatty acids (8). Staiger et al (16) reported that the muscle-derived ANGPTL4 was induced by fatty acids viaperoxisome proliferator activated receptor- $\gamma$ in humans. Stapleton et al (17) also reported that ANGPTL4 is expressed in human airway smooth muscle cells.

The current study investigated the expression of ANGPTL4 in muscle tissues, and evaluated the hypothesis that DSMA may mediate the depletion of ANGPTL4, and ANGPTL4 may have a protective role against DSMA. Indeed, the findings suggest that ANGPTL4 mRNA and protein were significantly increased in BYHWT-treated DSMA model rats, which suggests that the BYHWT significantly increased the levels of ANGPTL4 levels.

In order to confirm the role of $\mathrm{NF}-\kappa \mathrm{B}$ in the therapeutic effects of BYHWT, H\&E staining and TEM analysis were performed on samples of the anterior cervical muscle. Previous studies (18-20) demonstrated that there was obvious inflammation and serious ultrastructure changes in the muscle of the DSMA model. The present results indicated that the model group muscle exhibited serious inflammatory cell infiltration; however, the BYHWT treatment (Con-siRNA group) significantly decreased the inflammatory cell infiltration. Furthermore, when ANGPTL4 expression was suppressed using ANGPTL4-siRNA viral vector, the inflammatory cell infiltration was aggravated, and ultrastructural damaged was observed. These results also suggest that the BYHWT increases the expression of ANGPTL4, which may be critical for the improvement of the inflammation and ultrastructure, and ultimately improve the denervation-induced muscle atrophy.
In addition to the inflammation and ultrastructure changes, cell apoptosis may also participate in the processes of DSMA. Therefore, cell apoptosis of muscle cells was determined using a TUNEL assay. The result indicated that there was an increase in TUNEL-stained cells in the model group compared with the, normal control group, and the number of TUNEL-positive cells as significantly decreased in BYHWT treated groups, which suggest that BYHWT inhibits the apoptosis of muscle cells. These results are consistent with the previously published studies $(21,22)$.

In order to clarify the mechanism of the DSMA-associated cell apoptosis and the therapeutic effects of BYHWT, the inflammation-associated factor, $\mathrm{NF}-\kappa \mathrm{B}$ p65, and the atrophying skeletal muscle-associated factor, MURF1, were detected by western blot assay. Bodine et al (23) reported that MURF1 was induced in atrophying skeletal muscle, and exhibits reduced DSMA. Additionally, a previous study (20) demonstrated that the $\mathrm{NF}-\kappa \mathrm{B}$ signaling pathway regulates MURF1 expression when undergoing apoptotic assaults. The results of the current study demonstrated that the NF- $\kappa \mathrm{B}$ p65 and MURF1 expression were increased in the DSMA model, and were inhibited by treatment with BYHWT. Consistent with the previous studies (24-27), these results suggest that BYHWT may mediate its effects on the denervation-induced muscle atrophy by regulating MURF1 protein expression, induced via changes in the $\mathrm{NF}-\kappa \mathrm{B}$ signaling pathway.

Although the results of the current study are of interest, there are also certain limitations in this study. The ANGPTL4-siRNA did not produce an efficient to knockdown of ANPGTL4 expression. In future studies, a successful ANGPTL4 gene knockdown or gene knockout animal model should be established to investigate further.

In conclusion, BYHWT improves DSMA, potentially by increasing ANGPTL4 expression, involving NF- $\kappa \mathrm{B}$ signaling and MURF1.

\section{Acknowledgements}

This study was supported by the National Natural Science Foundation of China For Youth (grant no. 81302890), Natural Science Foundation of Colleges and Universities in Jiangsu Province (grant no. 13KJB360003), Natural Science Foundation 
of Jiangsu Province (grant no. BK2011816) and Natural Science Foundation of Doctoral point of Colleges and Universities in China (New Teachers; grant no. 20123237120004).

\section{References}

1. Tajrishi MM, Shin J, Hetman M and Kumar A: DNA methyltransferase $3 \mathrm{a}$ and mitogen-activated protein kinase signaling regulate the expression of fibroblast growth factor-inducible 14 (Fn14) during denervation-induced skeletal muscle atrophy. J Biol Chem 289: 19985-19999, 2014.

2. Bongers KS, Fox DK, Ebert SM, Kunkel SD, Dyle MC, Bullard SA, Dierdorff JM and Adams CM: Skeletal muscle denervation causes skeletal muscle atrophy through a pathway that involves both Gadd45a and HDAC4. Am J Physiol Endocrinol Metab 305: E907-E915, 2013.

3. Nagpal P, Plant PJ, Correa J, Bain A, Takeda M, Kawabe H, Rotin D, Bain JR and Batt JA: The ubiquitin ligase Nedd4-1 participates in denervation-induced skeletal muscle atrophy in mice. PLoS One 7: e46427, 2012.

4. Batt JA and Bain JR: Tibial nerve transection-a standardized model for denervation-induced skeletal muscle atrophy in mice. J Vis Exp: e50657, 2013.

5. Hsieh CH, Jeng SF, Wu CJ, Lu TH, Yang JC, Chen YC, Lin CJ and Rau CS: Altered expression of the microRNAS and their potential target genes in the soleus muscle after peripheral denervation and reinnervation in rats. J Trauma 70: 472-480, 2011.

6. Livak KJ and Schmittgen TD: Analysis of relative gene expression data using real-time quantitative PCR and the 2(-Delta Delta C(T)) method. Methods 25: 402-408, 2001.

7. Li G, Li QS, Li WB, Wei J, Chang WK, Chen Z, Qiao HY, Jia YW, Tian JH and Liang BS: miRNA targeted signaling pathway in the early stage of denervated fast and slow muscle atrophy. Neural Regen Res 11: 1293-1303, 2016.

8. van der Kolk BW, Goossens GH, Jocken JW, Kersten S and Blaak EE: Angiopoietin-like protein 4 and postprandial skeletal muscle lipid metabolism in overweight and obese prediabetics. J Clin Endocrinol Metab 101: 2332-2339, 2016.

9. Feingold KR, Shigenaga JK, Cross AS, Moser A and Grunfeld C: Angiopoietin like protein 4 expression is decreased in activated macrophages. Biochem Biophys Res Commun 421: 612-615, 2012.

10. Du D: Treatment of prolapse of lumbar intervertebral disc by tuina massotherapy combined with oral administration of buyang huanwu tang-a reprot of 75 cases. J Tradit Chin Med 27: 43-45, 2007.

11. Guo ZP, Huang MN, Liu AQ, Yuan YJ, Zhao JB and Mei XF: Buyang Huanwu decoction up-regulates Notch 1 gene expression in injured spinal cord. Neural Regen Res 10: 1321-1323, 2015.

12. Tiscornia G, Singer O, Ikawa M and Verma IM: A general method for gene knockdown in mice by using lentiviral vectors expressing small interfering RNA. Proc Natl Acad Sci USA 100: 1844-1848, 2003.

13. Tiscornia G, Singer O and Verma IM: Design and cloning of lentiviral vectors expressing small interfering RNAs. Nat Protoc 1: 234-240, 2006.

14. Liu X, Min Y, Gu W, Wang Y and Tian Y: Buyanghuanwu Tang therapy for neonatal rats with hypoxic ischemic encephalopathy. Int J Clin Exp Med 8: 18448-18454, 2015.
15. Jinglong T, Weijuan G, Jun L, Tao Q, Hongbo Z and Shasha L: The molecular and electrophysiological mechanism of buyanghuanwu decoction in learning and memory ability of vascular dementia rats. Brain Res Bull 99: 13-18, 2013.

16. Staiger H, Haas C, Machann J, Werner R, Weisser M, Schick F, Machicao F, Stefan N, Fritsche A and Häring HU: Muscle-derived angiopoietin-like protein 4 is induced by fatty acids via peroxisome proliferator-activated receptor (PPAR)-delta and is of metabolic relevance in humans. Diabetes 58: 579-589, 2009.

17. Stapleton CM, Joo JH, Kim YS, Liao G and Panettieri RA Jr and Jetten AM: Induction of ANGPTL4 expression in human airway smooth muscle cells by PMA through activation of PKC and MAPK pathways. Exp Cell Res 316: 507-516, 2010.

18. Chen HX, Tang SP, Gao FT, Xu JL, Jiang XP, Cao J, Fu GB, Sun K, Liu SZ and Shi W: Fibrosis, adipogenesis, and muscle atrophy in congenital muscular torticollis. Medicine (Baltimore) 93: e138, 2014.

19. Ohnishi Y, Iwatsuki K, Shinzawa K, Nakai Y, Ishihara M and Yoshimine T: Disuse muscle atrophy exacerbates motor neuronal degeneration caudal to the site of spinal cord injury. Neuroreport 23: 157-161, 2012.

20. Ohyama K, Koike H, Katsuno M, Takahashi M, Hashimoto R, Kawagashira Y, Iijima M, Adachi H, Watanabe $\mathrm{H}$ and Sobue G: Muscle atrophy in chronic inflammatory demyelinating polyneuropathy: A computed tomography assessment. Eur J Neurol 21: 1002-1010, 2014.

21. Meneses C, Morales MG, Abrigo J, Simon F, Brandan E and Cabello-Verrugio C: The angiotensin-(1-7)/Mas axis reduces myonuclear apoptosis during recovery from angiotensin II-induced skeletal muscle atrophy in mice. Pflugers Arch 467: 1975-1984, 2015.

22. Bargiela A, Cerro-Herreros E, Fernandez-Costa JM, Vilchez JJ, Llamusi B and Artero R: Increased autophagy and apoptosis contribute to muscle atrophy in a myotonic dystrophy type 1 Drosophila model. Dis Model Mech 8: 679-690, 2015.

23. Bodine SC, Latres E, Baumhueter S, Lai VK, Nunez L, Clarke BA, Poueymirou WT, Panaro FJ, Na E, Dharmarajan K, et al: Identification of ubiquitin ligases required for skeletal muscle atrophy. Science 294: 1704-1708, 2001.

24. Mittal A, Bhatnagar S, Kumar A, Lach-Trifilieff E, Wauters S, Li H, Makonchuk DY, Glass DJ and Kumar A: The TWEAK-Fn14 system is a critical regulator of denervation-induced skeletal muscle atrophy in mice. J Cell Biol 188: 833-849, 2010.

25. Cai D, Frantz JD, Tawa NE Jr, Melendez PA, Oh BC, Lidov HG, Hasselgren PO, Frontera WR, Lee J, Glass DJ and Shoelson SE: IKKbeta/NF-kappaB activation causes severe muscle wasting in mice. Cell 119: 285-298, 2004.

26. Mourkioti F, Kratsios P, Luedde T, Song YH, Delafontaine P, Adami R, Parente V, Bottinelli R, Pasparakis M and Rosenthal N: Targeted ablation of IKK2 improves skeletal muscle strength, maintains mass, and promotes regeneration. J Clin Invest 116: 2945-2954, 2006.

27. Macpherson PC, Wang X and Goldman D: Myogenin regulates denervation-dependent muscle atrophy in mouse soleus muscle. J Cell Biochem 112: 2149-2159, 2011.

This work is licensed under a Creative Commons Attribution-NonCommercial-NoDerivatives 4.0 International (CC BY-NC-ND 4.0) License. 